\title{
The Characterizations of WG Matrix and Its Generalized Cayley-Hamilton Theorem
}

\author{
Na Liu and Hongxing Wang \\ School of Mathematics and Physics, Guangxi Higher School Key Laboratory of Complex Systems and Intelligent Computing, \\ Guangxi University for Nationalities, Nanning 530006, China \\ Correspondence should be addressed to Hongxing Wang; winghongxing0902@163.com
}

Received 22 October 2021; Accepted 27 November 2021; Published 27 December 2021

Academic Editor: Efthymios G. Tsionas

Copyright ( $) 2021 \mathrm{Na}$ Liu and Hongxing Wang. This is an open access article distributed under the Creative Commons Attribution License, which permits unrestricted use, distribution, and reproduction in any medium, provided the original work is properly cited.

Based on the core-EP decomposition, we use the WG inverse, Drazin inverse, and other inverses to give some new characterizations of the WG matrix. Furthermore, we generalize the Cayley-Hamilton theorem for special matrices including the WG matrix. Finally, we give examples to verify these results.

\section{Introduction}

First, we use the following notations. Let $\mathbb{C}_{m, n}$ stand for the set of $m \times n$ complex matrices. The symbols $A^{*}, \mathscr{R}(A)$, $\operatorname{rk}(A)$, and $\operatorname{det}(A)$ represent the conjugate transpose, range, rank, and determinant of $A$, respectively. The smallest positive integer $k$ such that $\operatorname{rk}\left(A^{k+1}\right)=\operatorname{rk}\left(A^{k}\right)$ is called the index of $A \in \mathbb{C}_{m, n}$ and it is denoted by $\operatorname{Ind}(A)$. The Moore-Penrose inverse of $A \in \mathbb{C}_{m, n}$ is the unique matrix $X \in \mathbb{C}_{n, m}$ satisfying the following equations:

$$
\begin{gathered}
A X A=A, \\
X A X=X, \\
(A X)^{*}=A X, \\
(X A)^{*}=X A,
\end{gathered}
$$

and the unique matrix $X$ is denoted by $X=A^{\dagger}[1,2]$. Furthermore, we denote

$$
\begin{aligned}
& E_{A}=A A^{\dagger}, \\
& F_{A}=I_{n}-A^{\dagger} A .
\end{aligned}
$$

The Drazin inverse of $A \in \mathbb{C}_{n, n}$ is the unique matrix $X \in \mathbb{C}_{n, n}$ such that

$$
\begin{aligned}
A^{k} X A & =A^{k}, \\
X A X & =X, \\
A X & =X A,
\end{aligned}
$$

and the unique matrix $X$ is usually denoted by $X=A^{D}$, where $k=\operatorname{Ind}(A)[1,2]$. In particular, when $k=1, X$ is called the group inverse of $A$ and is denoted by $X=A^{\#}$. Therefore, we call it a group invertible matrix with index 1 . The symbol $\mathbb{C}_{n}^{C M}$ stands for the set of group invertible matrices in $\mathbb{C}_{n, n}$ :

$$
\mathbb{C}_{n}^{C M}=\operatorname{Ark}\left(A^{2}\right)=r k(A), A \in \mathbb{C}_{n, n} .
$$

Baksalary and Trenkler [3] defined the core inverse of a complex matrix with index 1 . Let $A \in \mathbb{C}_{n}^{\mathrm{CM}}$; the core inverse of $A$ is the unique matrix which satisfies the following equations:

$$
\begin{gathered}
A X=A A^{\oplus}, \\
\mathscr{R}(X) \subseteq \mathscr{R}(A),
\end{gathered}
$$

and it is denoted by $X=A^{\oplus}$. Subsequently, a variety of new generalized inverses have been established successively. Let $A \in \mathbb{C}_{n, n}$ with Ind $(A)=k$. The core-EP inverse of $A$ is the unique matrix $X \in \mathbb{C}_{n, n}$ satisfying $X A^{k+1}=A^{k}, X A X=X$, 
and $(A X)^{*}=A X$, and the core-EP inverse of $A$ is denoted by $A^{\oplus}[4]$; the B-T inverse of $A$ is the unique matrix $X \in \mathbb{C}_{n, n}$ satisfying $X=\left(A E_{A}\right)^{\dagger}$, and the $\mathrm{B}$-T inverse of $A$ is denoted by $A^{\diamond}$ [5]; the DMP inverse of $A$ is the unique matrix $X \in \mathbb{C}_{n, n}$ satisfying $X A X=X, X A=A^{D} A$, and $A^{k} X=A^{k} A^{\oplus}$, and the DMP inverse of $A$ is denoted by $X=A^{D, \dagger}$ [6]; the dual DMP inverse of $A$ is the unique matrix $X \in \mathbb{C}_{n, n}$ satisfying $X A X=X, A X=A A^{D}$, and $X A^{k}=A^{\oplus} A^{k}$, and the dual DMP inverse of $A$ is denoted by $A^{\dagger, D}[6]$; the CMP inverse of $A \in \mathbb{C}_{n, n}$ is the unique matrix $X \in \mathbb{C}_{n, n}$ satisfying $X A X=X$, $A X=A A^{D} A A^{\oplus}$, and $X A=A^{\oplus} A A^{D} A$, and the CMP inverse of $A \in \mathbb{C}_{n, n}$ is denoted by $A^{C, \dagger}$ [7]. It is easy to see that core-EP inverse and DMP inverse are both generalized core inverses, which are extensions of core inverse on square matrices without index constraint, and when $A \in \mathbb{C}_{n}^{\mathrm{CM}}, A^{\boxplus}=A^{D, \dagger}$ $=A^{\oplus}$.

Furthermore, Wang and Chen [8] proposed a generalized group inverse. Let $A \in \mathbb{C}_{n}^{\mathrm{CM}}$, if $X$ satisfies the following equations:

$$
\begin{aligned}
& A X^{2}=X, \\
& A X=A^{\oplus} A,
\end{aligned}
$$

where $X$ is called the WG inverse of $A$, and $X$ is unique. It is usually denoted by $X=A^{\circledR}$. By applying the definition, we can obtain $A^{\circledR} A A^{\circledR}=A^{\mathbb{1}}$ and $\mathscr{R}\left(A^{\mathbb{\Xi}}\right) \subseteq \mathscr{R}\left(A^{k}\right)$. It is noteworthy that $A^{\mathbb{1}}=A^{\#}$ and $A^{\#} \neq A^{\#}$, when $A \in \mathbb{C}_{n}^{\mathrm{CM}}$. Then, Ferryra et al. [9] extended the definition of WG inverse to the general matrix, defined the weighted WG inverse, and gave its expression, properties, and characterizations; Mosić and Zhang [10] established the weighted WG inverse of Hilbert space operator; Zhou et al. [11] generalized WG inverse to a proper $*$-ring and gave a new characterization of WG inverse; Zhou et al. [12] generalized $\mathrm{m}-\mathrm{WG}$ inverse to a unitary ring with involution and gave some properties of $\mathrm{m}-\mathrm{WG}$ inverse; Mosić and Stanimirović [13] gave new characterizations, limit representations, integral representations, and perturbation formulae of the WG inverse.

By applying the WG inverse, Wang and Liu [14] introduced the definition of WG matrix based on the properties and characterizations of WG inverse. Let $A \in \mathbb{C}_{n, n}$; if $A$ commutes with its WG inverse, $A$ is called WG matrix. The symbol $\mathbb{C}_{n}^{W G}$ stands for the set of WG matrices in $\mathbb{C}_{n, n}[14]$ :

$$
\mathbb{C}_{n}^{W G}=A A A^{\mathbb{(}}=A^{\circledR} A, \quad A \in \mathbb{C}_{n, n} .
$$

Subsequently, Yan et al. [15] investigated some new characterizations of weak group inverse by projection, the Bott-Duffin inverse, etc.

Matrix decomposition is very important, which not only functions as a significant role in every branch of mathematics but also has a wide range of applications in engineering. With the development of new generalized inverses, new research tools such as matrix decomposition and algorithm are also given. Wang established the core-EP decomposition of square matrix over complex fields [16]. CoreEP decomposition is one of the commonly used tools to study core-EP inverse and several new generalized inverses.
Lemma 1 (see [16], core-EP decomposition). Let $A \in \mathbb{C}_{n, n}$ with Ind $(A)=k$. Then, there exist $A_{1}$ and $A_{2}$, such that $A=$ $A_{1}+A_{2}$, where $A_{1} \in \mathbb{C}_{n}^{C M}, A_{2}^{k}=0$, and $A_{1}^{*} A_{2}=A_{2} A_{1}=0$. Furthermore,

$$
\begin{aligned}
& A_{1}=U\left[\begin{array}{ll}
T & S \\
0 & 0
\end{array}\right] U^{*}, \\
& A_{2}=U\left[\begin{array}{ll}
0 & 0 \\
0 & N
\end{array}\right] U^{*},
\end{aligned}
$$

where $U$ is a unitary matrix, $T \in \mathbb{C}_{r k\left(A^{k}\right), r k\left(A^{k}\right)}$ is nonsingular, and $N$ is nilpotent.

By applying the above decomposition, it is easy to verify

$$
A^{k}\left(A^{k}\right)^{\oplus}=U\left[\begin{array}{cc}
I_{r k}\left(A^{k}\right) & 0 \\
0 & 0
\end{array}\right] U^{*} .
$$

Lemma 2 (see [14]). Let $A \in \mathbb{C}_{n, n}$ with $\operatorname{Ind}(A)=k$, then $r k\left(A^{\circledR}\right)=r k\left(A^{D}\right)=r k\left(A^{k}\right)$ and

$$
\begin{aligned}
A^{\oplus} & =A_{1}^{\#}=U\left[\begin{array}{cc}
T^{-1} & T^{-2} S \\
0 & 0
\end{array}\right] U^{*}, \\
& =\left(A A^{\oplus} A\right)^{\#}=\left(A^{\oplus}\right)^{2} A=\left(A^{2}\right)^{\oplus} A \\
& =A^{k}\left(A^{k+2}\right)^{\oplus} A=\left(A^{2} E_{A^{k}}\right)^{\oplus} A .
\end{aligned}
$$

Lemma 3 (see $[8,14]$ ). Let $A \in \mathbb{C}_{n, n}$ be as in the form (8). Among $A \in \mathbb{C}_{n}^{W G},\left(A^{2}\right)^{\mathbb{1}}=\left(A^{\mathbb{1}}\right)^{2}, S N=0$, and $A A^{\mathbb{M}}=A^{\mathbb{\Xi}}$ $A$, any two of them are equivalent. If $A \in \mathbb{C}_{n}^{W G}$, then

$$
A^{\circledR}=A^{D}=\left(A^{k+1}\right)^{\circledR} A^{k}=\left(A^{t+1}\right)^{\oplus} A^{t}
$$

where $t$ is a positive integer and $k$ is the index of $A$.

Lemma 4 (see [16-20]). Let $A \in \mathbb{C}_{n, n}$ be as in the form [21], then

$$
\begin{aligned}
& \text { (1) } A^{\oplus}=U\left[\begin{array}{cc}
T^{*} \Delta & -T^{*} \Delta S N^{\oplus} \\
F_{N} S^{*} \Delta & N^{\oplus}-F_{N} S^{*} \Delta S N^{\oplus}
\end{array}\right] U^{*} ; \\
& \text { (2) } A^{D}=U\left[\begin{array}{cc}
T^{-1} & T^{-(k+1)} \widetilde{T} \\
0 & 0
\end{array}\right] U^{*} ; \\
& \text { (3) } A^{\oplus}=U\left[\begin{array}{cc}
T^{-1} & 0 \\
0 & 0
\end{array}\right] U^{*} ; \\
& \text { (4) } A^{\diamond}=U\left[\begin{array}{c}
T^{*} \triangle_{1} \\
\left(E_{N}-E_{\left.N^{\diamond}\right)} S^{*} \triangle N^{\diamond}-\left(E_{N^{-}}-E_{N^{\diamond}}\right) S^{*} \triangle_{1} S N^{\diamond}\right.
\end{array}\right] \\
& \text { (5) } A^{D, \dagger}=U\left[\begin{array}{cc}
T^{-1} & T^{-(k+1)} \widetilde{T} N N^{\oplus} \\
0 & 0
\end{array}\right] U^{*} ; \\
& \text { (6) } A^{\dagger, D}=U\left[\begin{array}{cc}
T^{*} \triangle & T^{*} \triangle T^{-k} \widetilde{T} \\
F_{N} S^{*} \triangle & F_{N} S^{*} \triangle T^{-k} \widetilde{T}
\end{array}\right] U^{*} ; \\
& \text { (7) } A^{C, \dagger}=U\left[\begin{array}{cc}
T^{*} \triangle & T^{*} \triangle T^{-k} \widetilde{T} N N^{\oplus} \\
F_{N} S^{*} \triangle & F_{N} S^{*} \triangle T^{-k} \widetilde{T} N N^{\oplus}
\end{array}\right] U^{*},
\end{aligned}
$$


where $\triangle_{1}=\left(T T^{*}+S\left(E_{N}-E_{N^{\diamond}} S^{*}\right)\right)^{-1}, \quad k=\operatorname{Ind}(A)$, $\widetilde{T}=\sum_{i=0}^{k-1} T^{i} S N^{k-1-i}$, and $\triangle=\left(T T^{*}+S F_{N} S^{*}\right)^{-1}$.

The classical Cayley-Hamilton theorem is one of the most important theorems in matrix theory. On the basis of the classical Cayley-Hamilton theorem, mathematicians established the rectangular matrix, block matrix, pair of block matrix, and other matrices as well as more generalized Cayley-Hamilton theorem for generalized inverse matrices. They also gave application of generalized Cayley-Hamilton theorem in several control systems [22-25]. In [26], Wang, Chen, and Yan gave the generalized Cayley-Hamilton theorem of core-EP inverse matrix and DMP inverse matrix by core-EP decomposition, and the characteristic polynomial equations of core-EP inverse matrix and DMP inverse matrix were also discussed. In [2, 27], the researchers studied the applications of generalized Cayley-Hamilton theorems in generalized inverses such as Drazin inverse and Moore-Penrose inverse. Based on the above researches, this paper will focus on the WG matrix, the equivalent characterizations of WG matrix, and the generalized Cayley-Hamilton theorem for special matrices including WG matrix.

\section{Some Characterizations of WG Matrix}

In [14], the definition and characterizations of WG matrix are given through the commutativity of matrix and WG inverse. In [15], Yan et al. investigated some new characterizations of WG matrix.

Theorem 1 (see [15]). Let $A \in \mathbb{C}_{n, n}$ with $\operatorname{Ind}(A)=k$, then the following conditions are equivalent:
(1) $A \in \mathbb{C}_{n}^{W G}$;
(2) $A^{k} A^{\oplus}=A^{k} A^{\oplus}$;
(3) $A^{k} A^{\oplus}=A^{k} A^{D, \dagger}$;
(4) $A^{\oplus} A^{k}=A^{k} A^{\boxplus ;}$
(5) $A A^{D}=A^{\oplus} A$;
(6) $A A^{D}=A A^{\circledR}$;
(7) $A^{\oplus} A=A^{D, \dagger} A$.

It is pointed out that the set of group invertible matrices is a subset of WG matrices set. Special matrices such as WG matrix, group matrix, $\mathrm{EP}, i$-EP, and $k$-EP matrix have rich intersection [14]. In this section, we will mainly apply core-EP decomposition to study the characterization of WG matrix.

Theorem 2. Let $A \in \mathbb{C}_{n, n}$ with Ind $(A)=k$, then the following conditions are equivalent:

(1) $S N=0$, where $S$ and $N$ are as in the form [21];

(2) $A A^{\circledR}=A^{\circledR} A$;

(3) $\left(A^{2}\right)^{\mathbb{1}}=\left(A^{\mathbb{Q}}\right)^{2}$;

(4) $A^{\circledR}=A^{D}$;

(5) $A^{\oplus} A$ commutes with $A^{\oplus} A^{2}$;

(6) $U A U^{*}$ is a WG matrix for any unitary matrix $U$;

(7) A commutes with $A A^{\circledR}$;
(8) A commutes with $A^{\oplus} A$;

(9) $A A^{\oplus} A=A A^{\circledR} A$;

(10) $A^{\circledR}$ commutes with $A^{\circledR} A$;

(11) $A^{k}\left(A^{k}\right)^{\oplus} A^{2}=\left(A^{k}\left(A^{k}\right)^{\oplus} A\right)^{2}$;

(12) $A A^{\oplus} A^{2}=\left(A A^{\oplus} A\right)^{2}$;

(13) $A^{\circledR} A=A^{\circledR} A^{k}\left(A^{k}\right)^{\oplus} A$;

(14) $A^{\circledR} A=A^{\circledR} A A^{\oplus} A$;

(15) $A\left(A^{\circledR}\right)^{2} A=A A^{\circledR}$;

(16) $A^{\circledR} A^{2} A^{\circledR}=A^{\circledR} A$;

(17) $A\left(A^{2}\right)^{\circledR}=A^{\boxplus}$;

(18) $A\left(A^{2}\right)^{\circledR}=A A^{\oplus} A^{\circledR}$;

(19) $A\left(A^{2}\right)^{\circledR}=A A^{D, \dagger} A^{(}$;

(20) $\left(A A^{\mathbb{( 1}}\right)^{2}=\left(A^{\mathbb{\circledR}}\right)^{2} A A^{\mathbb{1}} A^{2}$;

(21) $\left(A^{\circledR} A\right)^{2}=A^{2}\left(A^{\circledR}\right)^{2}$;

(22) $A A^{\circledR}$ commutes with $A^{\circledR} A$;

(23) $A A^{\circledR} A=A^{k}\left(A^{k}\right)^{\oplus} A$;

(24) $\left(A^{\mathbb{(}(1)}\right)^{2} A=A^{\mathbb{1}}$;

(25) $\left(A^{\circledR}\right)^{2} A=A^{D, \dagger} A A^{\circledR}$;

(26) $\left(A^{\circledR}\right)^{2} A=A A^{\dagger, D} A^{\circledR}$;

(27) $\left(A^{\circledR}\right)^{2} A=A A^{C, \dagger} A^{\circledR}$;

(28) $A^{\circledR} A^{D} A=A^{\circledR}$;

(29) $A^{\circledR}$ commutes with $A A^{D}$;

(30) $A A^{D}\left(I-A A^{\oplus}\right) A=0$;

(31) $A^{k}\left(I-A A^{\oplus}\right) A=0$;

(32) $A A^{D, \dagger}\left(I-A A^{\oplus}\right) A=0$;

(33) $A^{\dagger, D}\left(I-A A^{\oplus}\right) A=0$;

(34) $A^{C, \dagger}\left(I-A A^{\oplus}\right) A=0$;

(35) $A^{\oplus} A^{2}=A^{2} A^{\circledR}$;

(36) $A^{2} A^{D}=A A^{\oplus} A$;

Proof. From [14], we know that Conditions (1)-(5) are equivalent.

Let $U$ be a unitary matrix, then $\left(U A U^{*}\right)^{\circledR}=U A^{\circledR} U^{*}$ and

$$
\left\{\begin{array}{l}
U A A^{\circledR} U^{*}=U A U^{*} U A^{\circledR} U^{*}=\left(U A U^{*}\right)\left(U A U^{*}\right)^{\circledR}, \\
U A^{\circledR} A U^{*}=U A^{\circledR} U^{*} U A U^{*}=\left(U A U^{*}\right)^{\circledR}\left(U A U^{*}\right) .
\end{array}\right.
$$

Thus, Conditions (2) and (6) are equivalent.

Let the core-EP decomposition of $A$ be as in the form [21]. By using Lemma 3, we obtain

$$
\begin{aligned}
A A^{\circledR} & =U\left[\begin{array}{ll}
T & S \\
0 & N
\end{array}\right]\left[\begin{array}{cc}
T^{-1} & T^{-2} S \\
0 & 0
\end{array}\right] U^{*}=U\left[\begin{array}{cc}
I_{r k}\left(A^{k}\right) & T^{-1} S \\
0 & 0
\end{array}\right] U^{*}, \\
A^{\circledR} A & =U\left[\begin{array}{cc}
T^{-1} & T^{-2} S \\
0 & 0
\end{array}\right]\left[\begin{array}{cc}
T & S \\
0 & N
\end{array}\right] U^{*} \\
& =U\left[\begin{array}{cc}
I_{r k\left(A^{k}\right)} & T^{-1} S+T^{-2} S N \\
0 & 0
\end{array}\right] U^{*} .
\end{aligned}
$$


Applying [21, 27], we have

$$
\begin{aligned}
& A A A^{\circledR}=U\left[\begin{array}{cc}
T & S \\
0 & N
\end{array}\right]\left[\begin{array}{cc}
I_{r k}\left(A^{k}\right) & T^{-1} S \\
0 & 0
\end{array}\right] U^{*}=U\left[\begin{array}{ll}
T & S \\
0 & 0
\end{array}\right] U^{*}, \\
& A A^{\circledR} A=U\left[\begin{array}{cc}
I_{r k}\left(A^{k}\right) & T^{-1} S \\
0 & 0
\end{array}\right]\left[\begin{array}{cc}
T & S \\
0 & N
\end{array}\right] U^{*}=U\left[\begin{array}{cc}
T & S+T^{-1} S N S \\
0 & 0
\end{array}\right] U^{*} .
\end{aligned}
$$

By comparing $[4,7]$, we can get

$$
S N=0 \Longleftrightarrow A A A^{\circledR}=A A^{\circledR} A \text {. }
$$

$$
A A^{\oplus}=U\left[\begin{array}{cc}
T & S \\
0 & N
\end{array}\right]\left[\begin{array}{cc}
T^{-1} & 0 \\
0 & 0
\end{array}\right] U^{*}=U\left[\begin{array}{cc}
I_{r k}\left(A^{k}\right) & 0 \\
0 & 0
\end{array}\right] U^{*},
$$

Thus, Conditions (1) and (7) are equivalent.

By Lemma 4, we obtain

$$
A^{\oplus} A=U\left[\begin{array}{cc}
T^{-1} & 0 \\
0 & 0
\end{array}\right]\left[\begin{array}{cc}
T & S \\
0 & N
\end{array}\right] U^{*}=U\left[\begin{array}{cc}
I_{r k}\left(A^{k}\right) & T^{-1} S \\
0 & 0
\end{array}\right] U^{*} .
$$

By applying $[10,13]$, we have

$$
\begin{aligned}
& A A^{\oplus} A=U\left[\begin{array}{cc}
I_{r k\left(A^{k}\right)} & 0 \\
0 & 0
\end{array}\right]\left[\begin{array}{cc}
T & S \\
0 & N
\end{array}\right] U^{*}=U\left[\begin{array}{ll}
T & S \\
0 & 0
\end{array}\right] U^{*}, \\
& A^{\oplus} A A=U\left[\begin{array}{cc}
I_{r k}\left(A^{k}\right) & T^{-1} S \\
0 & 0
\end{array}\right]\left[\begin{array}{cc}
T & S \\
0 & N
\end{array}\right] U^{*}=U\left[\begin{array}{cc}
T & S+T^{-1} S N \\
0 & 0
\end{array}\right] U^{*} .
\end{aligned}
$$

From what has been discussed above, we can surely come to the conclusion that

$$
S N=0 \Longleftrightarrow A A^{\oplus} A=A^{\oplus} A A .
$$

Thus, Conditions (1) and (8) are equivalent. Because of $[7,16]$, we can get

$$
S N=0 \Longleftrightarrow A A^{\oplus} A=A A^{\circledR} A .
$$

Thus, Conditions (1) and (9) are equivalent.

From [6, 23], we obtain

$$
\begin{aligned}
A^{\circledR} A^{\circledR} A & =U\left[\begin{array}{cc}
T^{-1} & T^{-2} S \\
0 & 0
\end{array}\right]\left[\begin{array}{cc}
I_{r k}\left(A^{k}\right) & T^{-1} S+T^{-2} S N \\
0 & 0
\end{array}\right] U^{*} \\
& =U\left[\begin{array}{cc}
T^{-1} & T^{-2} S+T^{-3} S N \\
0 & 0
\end{array}\right] U^{*}, \\
A^{\circledR} A A^{\circledR} & =U\left[\begin{array}{cc}
I_{r k}\left(A^{k}\right) & T^{-1} S+T^{-2} S N \\
0 & 0
\end{array}\right]\left[\begin{array}{cc}
T^{-1} & T^{-2} S \\
0 & 0
\end{array}\right] U^{*} \\
& =U\left[\begin{array}{cc}
T^{-1} & T^{-2} S \\
0 & 0
\end{array}\right] U^{*} .
\end{aligned}
$$

By comparing the above equations, we have

$$
S N=0 \Longleftrightarrow A^{\circledR} A^{\circledR} A=A^{\circledR} A A^{\circledR} .
$$

Thus, Conditions (1) and (10) are equivalent. By using [19], we can get

$$
\begin{aligned}
A^{k}\left(A^{k}\right)^{\oplus} A^{2} & =U\left[\begin{array}{cc}
I_{r k\left(A^{k}\right)} & 0 \\
0 & 0
\end{array}\right]\left[\begin{array}{cc}
T & S \\
0 & N
\end{array}\right]\left[\begin{array}{cc}
T & S \\
0 & N
\end{array}\right] U^{*} \\
& =U\left[\begin{array}{cc}
T^{2} & T S+S N \\
0 & 0
\end{array}\right] U^{*}, \\
\left(A^{k}\left(A^{k}\right)^{\oplus} A\right)^{2} & =U\left[\begin{array}{cc}
I_{r k\left(A^{k}\right)} & 0 \\
0 & 0
\end{array}\right]\left[\begin{array}{cc}
T & S \\
0 & N
\end{array}\right] \\
& \cdot\left[\begin{array}{cc}
I_{r k}\left(A^{k}\right) & 0 \\
0 & 0
\end{array}\right]\left[\begin{array}{cc}
T & S \\
0 & N
\end{array}\right] U^{*} \\
& =U\left[\begin{array}{cc}
T^{2} & T S \\
0 & 0
\end{array}\right] U^{*} .
\end{aligned}
$$

By applying the above equations, we obtain

$$
S N=0 \Longleftrightarrow A^{k}\left(A^{k}\right)^{\oplus} A^{2}=\left(A^{k}\left(A^{k}\right)^{\oplus} A\right)^{2} .
$$

Thus, Conditions (1) and (11) are equivalent. Applying [10, 13], we have 


$$
\begin{aligned}
A A^{\oplus} A^{2} & =U\left[\begin{array}{cc}
I_{r k\left(A^{k}\right)} & 0 \\
0 & 0
\end{array}\right]\left[\begin{array}{cc}
T & S \\
0 & N
\end{array}\right]\left[\begin{array}{cc}
T & S \\
0 & N
\end{array}\right] U^{*}=U\left[\begin{array}{cc}
T^{2} & T S+S N \\
0 & 0
\end{array}\right] U^{*}, \\
\left(A A^{\oplus} A\right)^{2} & =U\left[\begin{array}{cc}
T & S \\
0 & N
\end{array}\right]\left[\begin{array}{cc}
I_{r k\left(A^{k}\right)} T^{-1} S \\
0 & 0
\end{array}\right]\left[\begin{array}{cc}
T & S \\
0 & N
\end{array}\right]\left[\begin{array}{cc}
I_{r k}\left(A^{k}\right) & T^{-1} S \\
0 & 0
\end{array}\right] U^{*}=U\left[\begin{array}{cc}
T^{2} & T S \\
0 & 0
\end{array}\right] U^{*} .
\end{aligned}
$$

By comparing the above equations, we can get

$$
S N=0 \Longleftrightarrow A A^{\oplus} A^{2}=\left(A A^{\oplus} A\right)^{2} .
$$

Thus, Conditions (1) and (12) are equivalent. By [19], we obtain

$$
\begin{aligned}
A^{\circledR} A^{k}\left(A^{k}\right)^{\oplus} A & =U\left[\begin{array}{cc}
T^{-1} & T^{-2} S \\
0 & 0
\end{array}\right]\left[\begin{array}{cc}
I_{\mathrm{rk}\left(A^{k}\right)} & 0 \\
0 & 0
\end{array}\right]\left[\begin{array}{cc}
T & S \\
0 & N
\end{array}\right] U^{*} \\
& =U\left[\begin{array}{cc}
I_{\mathrm{rk}\left(A^{k}\right)} & T^{-1} S \\
0 & 0
\end{array}\right] U^{*} .
\end{aligned}
$$

From the above equation and [6], we can get

$$
\mathrm{SN}=0 \Longleftrightarrow A^{\mathrm{W}} A=A^{\mathrm{W}} A^{k}\left(A^{k}\right)^{\oplus} A .
$$

Thus, Conditions (1) and (13) are equivalent.

By applying $[13,23]$, we have

$$
\begin{aligned}
A^{\circledR} A A^{\oplus} A & =U\left[\begin{array}{cc}
T^{-1} & T^{-2} S \\
0 & 0
\end{array}\right]\left[\begin{array}{cc}
I_{\mathrm{rk}\left(A^{k}\right)} & 0 \\
0 & 0
\end{array}\right]\left[\begin{array}{cc}
T & S \\
0 & N
\end{array}\right] U^{*} \\
& =U\left[\begin{array}{cc}
I_{\mathrm{rk}\left(A^{k}\right)} & T^{-1} S \\
0 & 0
\end{array}\right] U^{*} .
\end{aligned}
$$

By comparing the above equation and [6], we can get

$$
\mathrm{SN}=0 \Longleftrightarrow A^{\circledR} A=A^{\circledR} A A^{\oplus} A .
$$

Thus, Conditions (1) and (14) are equivalent. Because of $[6,27]$, we obtain

$$
\begin{aligned}
A\left(A^{\boxplus}\right)^{2} A= & U\left[\begin{array}{cc}
I_{r k}\left(A^{k}\right) & T^{-1} S \\
0 & 0
\end{array}\right] \\
& \cdot\left[\begin{array}{cc}
I_{r k}\left(A^{k}\right) & T^{-1} S+T^{-2} S N \\
0 & 0
\end{array}\right] U^{*} \\
= & U\left[\begin{array}{cc}
I_{\operatorname{rk}\left(A^{k}\right)} & T^{-1} S+T^{-2} S N \\
0 & 0
\end{array}\right] U^{*} .
\end{aligned}
$$

By the above equation and [27], we have

$$
\mathrm{SN}=0 \Longleftrightarrow A\left(A^{\circledR}\right)^{2} A=A A^{\circledR} \text {. }
$$

Thus, Conditions (1) and (15) are equivalent. From [6, 27], we can get

$$
\begin{aligned}
A^{\circledR} A^{2} A^{\circledR}= & U\left[\begin{array}{cc}
I_{\mathrm{rk}\left(A^{k}\right)} & T^{-1} S+T^{-2} S N \\
0 & 0
\end{array}\right] \\
& \cdot\left[\begin{array}{cc}
I_{\mathrm{rk}\left(A^{k}\right)} & T^{-1} S \\
0 & 0
\end{array}\right] U^{*} \\
= & U\left[\begin{array}{cc}
I_{\mathrm{rk}\left(A^{k}\right)} & T^{-1} S \\
0 & 0
\end{array}\right] U^{*} .
\end{aligned}
$$

By comparing the above equation and [6], we obtain

$$
\mathrm{SN}=0 \Longleftrightarrow A^{\circledR} A^{2} A^{\circledR}=A^{\circledR} A \text {. }
$$

Hence, Conditions (1) and (16) are equivalent. By using Lemma 2, we have

$$
\begin{aligned}
A\left(A^{2}\right)^{\mathrm{W}} & =U\left[\begin{array}{cc}
T & S \\
0 & N
\end{array}\right]\left[\begin{array}{cc}
T^{-2} & T^{-4}(\mathrm{TS}+\mathrm{SN}) \\
0 & 0
\end{array}\right] U^{*} \\
& =U\left[\begin{array}{cc}
T^{-1} & T^{-2} S+T^{-3} \mathrm{SN} \\
0 & 0
\end{array}\right] U^{*} .
\end{aligned}
$$

From the above equation and [23], we obtain

$$
\mathrm{SN}=0 \Longleftrightarrow A\left(A^{2}\right)^{\mathrm{W}}=A^{\mathrm{W}} \text {. }
$$

Thus, Conditions (1) and (17) are equivalent.

By applying Lemma 4, we can get

$$
\begin{aligned}
A A^{\oplus} & =U\left[\begin{array}{cc}
T & S \\
0 & N
\end{array}\right]\left[\begin{array}{cc}
T^{*} \triangle & -T^{*} \triangle \mathrm{SN}^{\oplus} \\
F_{N} S^{*} \triangle & N^{\oplus}-F_{N} S^{*} \triangle \mathrm{SN}^{\oplus}
\end{array}\right] U^{*} \\
& =U\left[\begin{array}{cc}
I_{\mathrm{rk}}\left(A^{k}\right) & 0 \\
0 & N N^{\oplus}
\end{array}\right] U^{*} .
\end{aligned}
$$

By (41), we obtain

$$
\begin{aligned}
A A^{\oplus} A^{\mathrm{W}} & =U\left[\begin{array}{cc}
I_{\mathrm{rk}\left(A^{k}\right)} & 0 \\
0 & N N^{\oplus}
\end{array}\right]\left[\begin{array}{cc}
T^{-1} & T^{-2} S \\
0 & 0
\end{array}\right] U^{*} \\
& =U\left[\begin{array}{cc}
T^{-1} & T^{-2} S \\
0 & 0
\end{array}\right] U^{*} .
\end{aligned}
$$

By applying the above formula and (39), we have $S N=0$ if and only if $A\left(A^{2}\right)^{\circledR}=A A^{\oplus} A^{\circledR}$. Thus, Conditions (1) and (18) are equivalent.

By applying Lemma 4, we can get

$$
\begin{aligned}
A A^{D, \dagger} & =U\left[\begin{array}{cc}
T & S \\
0 & N
\end{array}\right]\left[\begin{array}{cc}
T^{-1} & T^{-(k+1)} \widetilde{T} N N^{\oplus} \\
0 & 0
\end{array}\right] U^{*} \\
& =U\left[\begin{array}{cc}
I_{\mathrm{rk}}\left(A^{k}\right) & T^{-k} \widetilde{T} N N^{\oplus} \\
0 & 0
\end{array}\right] U^{*} .
\end{aligned}
$$


Because of (43), we obtain

$$
\begin{aligned}
A A^{D, \dagger} A^{\circledR} & =U\left[\begin{array}{cc}
I_{\mathrm{rk}\left(A^{k}\right)} & T^{-k} \widetilde{T} N N^{\oplus} \\
0 & 0
\end{array}\right]\left[\begin{array}{cc}
T^{-1} & T^{-2} S \\
0 & 0
\end{array}\right] U^{*} \\
& =U\left[\begin{array}{cc}
T^{-1} & T^{-2} S \\
0 & 0
\end{array}\right] U^{*} .
\end{aligned}
$$

By comparing the above formula and (39), we obtain $S N=0$ if and only if $A\left(A^{2}\right)^{\mathbb{1}}=A A^{D, \dagger} A^{\mathbb{Q}}$. Thus, Conditions (1) and (19) are equivalent.

Applying [6, 27], we have

$$
\begin{aligned}
\left(A A^{\circledR}\right)^{2} & =U\left[\begin{array}{cc}
I_{\mathrm{rk}}\left(A^{k}\right) & T^{-1} S \\
0 & 0
\end{array}\right]^{2} U^{*}=U\left[\begin{array}{cc}
I_{\mathrm{rk}}\left(A^{k}\right) & T^{-1} S \\
0 & 0
\end{array}\right] U^{*}, \\
\left(A^{\circledR}\right)^{2} A A^{\circledR} A^{2} & =U\left[\begin{array}{cc}
T^{-1} & T^{-2} S \\
0 & 0
\end{array}\right]^{2}\left[\begin{array}{cc}
I_{\mathrm{rk}}\left(A^{k}\right) & T^{-1} S \\
0 & 0
\end{array}\right]\left[\begin{array}{cc}
T & S \\
0 & N
\end{array}\right]^{2} U^{*}, \\
& =U\left[\begin{array}{cc}
I_{\mathrm{rk}\left(A^{k}\right)} & T^{-1} S+T^{-2} S N+T^{-3} S N^{2} \\
0 & 0
\end{array}\right] U^{*} .
\end{aligned}
$$

If $S N=0$, then $\left(A A^{\circledR}\right)^{2}=\left(A^{\circledR}\right)^{2} A A^{\circledR} A^{2}$. Conversely, let $\left(A A^{\circledR}\right)^{2}=\left(A^{\circledR}\right)^{2} A A^{\circledR} A^{2}$; we get $T^{-1} S+T^{-2} S N+T^{-3}$ $S N^{2}=T^{-1} S$, that is, $T S N+S N^{2}=0$.

If the index of $A$ is equal to 1 , then $N=0$, that is, $S N=0$. If the index of $A$ is equal to 2 , then $N^{2}=0$. From $T S N+S N^{2}=0$, we have $T S N=0$. Since $T$ is invertible, we obtain $S N=0$. Let the index of $A$ be more than or equal to 3, then $N^{k}=0$ and $N^{k-1} \neq 0$. If $T S N+S N^{2}=0$ postmultiplication by $N^{k-2}$, then $T S N^{k-1}+S N^{k}=0$. Since $T$ is invertible and $N^{k}=0$, then $S N^{k-1}=0$. Furthermore, $T S N+$ $S N^{2}=0$ postmultiplication by $N^{k-3}$, then $T S N^{k-2}+S N^{k-1}$ $=0$. Since $T$ is invertible and $N^{k-1}=0$, then $S N^{k-2}=0$. By repeating the process $k-1$ times, we have $S N=0$.

From what has been discussed above, $S N=0$ if and only if $\left(A A^{\circledR}\right)^{2}=\left(A^{\circledR}\right)^{2} A A^{\circledR} A^{2}$. Thus, Conditions (1) and (20) are equivalent.

By $[6,21,23]$, we obtain

$$
\begin{aligned}
\left(A^{\circledR} A\right)^{2} & =U\left[\begin{array}{cc}
I_{\mathrm{rk}\left(A^{k}\right)} & T^{-1} S+T^{-2} S N \\
0 & 0
\end{array}\right]^{2} U^{*} \\
& =U\left[\begin{array}{cc}
I_{\mathrm{rk}\left(A^{k}\right)} & T^{-1} S+T^{-2} S N \\
0 & 0
\end{array}\right] U^{*}, \\
A^{2}\left(A^{\circledR}\right)^{2} & =U\left[\begin{array}{cc}
T & S \\
0 & N
\end{array}\right]^{2}\left[\begin{array}{cc}
T^{-1} & T^{-2} S \\
0 & 0
\end{array}\right]^{2} U^{*} \\
& =U\left[\begin{array}{cc}
I_{\mathrm{rk}}\left(A^{k}\right) & T^{-1} S \\
0 & 0
\end{array}\right] U^{*} .
\end{aligned}
$$

By comparing the above equations, we can get

$$
S N=0 \Longleftrightarrow\left(A^{\circledR} A\right)^{2}=A^{2}\left(A^{\circledR}\right)^{2} .
$$

Thus, Conditions (1) and (21) are equivalent.
By applying (35) and (37), we have $S N=0$ if and only if $\left(A A^{\mathrm{W}}\right)\left(A^{\mathrm{W}} A\right)=\left(A^{\mathrm{W}} A\right)\left(A A^{\mathrm{W}}\right)$. Thus, Conditions (1) and (22) are equivalent.

Because of [19], we have

$$
A^{k}\left(A^{k}\right)^{\oplus} A=U\left[\begin{array}{cc}
I_{\mathrm{rk}\left(A^{k}\right)} & 0 \\
0 & 0
\end{array}\right]\left[\begin{array}{cc}
T & S \\
0 & N
\end{array}\right] U^{*}=U\left[\begin{array}{ll}
T & S \\
0 & 0
\end{array}\right] U^{*}
$$

By comparing the above equation and [7], we obtain $A A^{\circledR} A=A^{k}\left(A^{k}\right)^{\oplus} A . S N=0$ is equivalent to $A A^{\circledR} A=A^{k}$ $\left(A^{k}\right)^{\oplus} A$. Hence, Conditions (1) and (23) are equivalent.

From [2, 23], we have $S N=0$ if and only if $\left(A^{\circledR}\right)^{2} A=$ $A^{\circledR}$. Thus, Conditions (1) and (24) are equivalent.

By using Lemma 4 , we obtain

$$
\begin{aligned}
A^{D, \dagger} A & =U\left[\begin{array}{cc}
T^{-1} & T^{-(k+1)} \widetilde{T} N N^{\oplus} \\
0 & 0
\end{array}\right]\left[\begin{array}{cc}
T & S \\
0 & N
\end{array}\right] U^{*} \\
& =U\left[\begin{array}{cc}
I_{\mathrm{rk}}\left(A^{k}\right) & T^{-1} S+T^{-(k+1)} \widetilde{T} N \\
0 & 0
\end{array}\right] U^{*} .
\end{aligned}
$$

By using (49), we have

$$
\begin{aligned}
A^{D, \dagger} A A^{\circledR} & =U\left[\begin{array}{cc}
I_{\mathrm{rk}\left(A^{k}\right)} & T^{-1} S+T^{-(k+1)} \widetilde{T} N \\
0 & 0
\end{array}\right]\left[\begin{array}{cc}
T^{-1} & T^{-2} S \\
0 & 0
\end{array}\right] U^{*} \\
& =U\left[\begin{array}{cc}
T^{-1} & T^{-2} S \\
0 & 0
\end{array}\right] U^{*}=A^{\circledR} .
\end{aligned}
$$

By comparing the above formula and [2], we can get $S N=0$ if and only if $\left(A^{\circledR}\right)^{2} A=A^{D, \dagger} A A^{\circledR}$. Thus, Conditions (1) and (25) are equivalent.

By Lemma 4, we obtain 


$$
\begin{aligned}
A A^{\dagger, D} & =U\left[\begin{array}{cc}
T & S \\
0 & N
\end{array}\right]\left[\begin{array}{cc}
T^{*} \triangle & T^{*} \triangle T^{-k} \widetilde{T} \\
F_{N} S^{*} \triangle & F_{N} S^{*} \triangle T^{-k} \widetilde{T}
\end{array}\right] U^{*} \\
& =U\left[\begin{array}{cc}
I_{\mathrm{rk}\left(A^{k}\right)} & T^{-k} \widetilde{T} \\
0 & 0
\end{array}\right] U^{*} .
\end{aligned}
$$

By applying (51), we have

$$
\begin{aligned}
A A^{\dagger, D} A^{\circledR} & =U\left[\begin{array}{cc}
I_{\mathrm{rk}\left(A^{k}\right)} & T^{-k} \widetilde{T} \\
0 & 0
\end{array}\right]\left[\begin{array}{cc}
T^{-1} & T^{-2} S \\
0 & 0
\end{array}\right] U^{*} \\
& =U\left[\begin{array}{cc}
T^{-1} & T^{-2} S \\
0 & 0
\end{array}\right] U^{*}=A^{\circledR} .
\end{aligned}
$$

Applying the above formula and [2], we can get $S N=0$ if and only if $\left(A^{\circledR}\right)^{2} A=A A^{\dagger, D} A^{\circledR}$. Hence, Conditions (1) and (26) are equivalent.

Because of Lemma 4, we obtain

$$
\begin{aligned}
A A^{C, \dagger} & =U\left[\begin{array}{cc}
T & S \\
0 & N
\end{array}\right]\left[\begin{array}{cc}
T^{*} \triangle & T^{*} \triangle T^{-k} \widetilde{T} N N^{\oplus} \\
F_{N} S^{*} \triangle & F_{N} S^{*} \triangle T^{-k} \widetilde{T} N N^{\oplus}
\end{array}\right] U^{*} \\
& =U\left[\begin{array}{cc}
I_{\mathrm{rk}\left(A^{k}\right)} & T^{-k} \widetilde{T} N N^{\oplus} \\
0 & 0
\end{array}\right] U^{*} .
\end{aligned}
$$

From (53), we have

$$
\begin{aligned}
A A^{C, \dagger} A^{\circledR} & =U\left[\begin{array}{cc}
I_{\mathrm{rk}\left(A^{k}\right)} & T^{-k} \widetilde{T} N N^{\oplus} \\
0 & 0
\end{array}\right]\left[\begin{array}{cc}
T^{-1} & T^{-2} S \\
0 & 0
\end{array}\right] U^{*} \\
& =U\left[\begin{array}{cc}
T^{-1} & T^{-2} S \\
0 & 0
\end{array}\right] U^{*}=A^{\boxplus} .
\end{aligned}
$$

By comparing the above formula and [2], we can get $S N=0$ if and only if $\left(A^{\circledR}\right)^{2} A=A A^{C, \dagger} A^{\circledR}$. Hence, Conditions (1) and (27) are equivalent.

By using Lemma 1 and Lemma 4, we obtain

$$
\begin{aligned}
A^{D} A & =U\left[\begin{array}{cc}
T^{-1} & T^{-(k+1)} \widetilde{T} \\
0 & 0
\end{array}\right]\left[\begin{array}{cc}
T & S \\
0 & N
\end{array}\right] U^{*} \\
& =U\left[\begin{array}{cc}
I_{\operatorname{rk}}\left(A^{k}\right) & T^{-1} S+T^{-(k+1)} \widetilde{T} N \\
0 & 0
\end{array}\right] U^{*} .
\end{aligned}
$$

By using the above formula, we have

$$
\begin{aligned}
A^{\circledR} A^{D} A & =U\left[\begin{array}{cc}
T^{-1} & T^{-2} S \\
0 & 0
\end{array}\right]\left[\begin{array}{cc}
I_{\mathrm{rk}\left(A^{k}\right)} & T^{-1} S+T^{-(k+1)} \widetilde{T} N \\
0 & 0
\end{array}\right] U^{*} \\
& =U\left[\begin{array}{cc}
T^{-1} & T^{-2} S+T^{-(k+2)} \widetilde{T} N \\
0 & 0
\end{array}\right] U^{*} .
\end{aligned}
$$

If $S N=0$, we can easily prove that $A^{\circledR} A^{D} A=A^{\circledR}$. Conversely, if the index of $A$ is equal to 1 or 2, then obviously $S N=0$. Let the index of $A$ be more than or equal to 3 ; we get $T^{-2} S+T^{-(k+2)}\left(\sum_{i=0}^{k-1} T^{i} S N^{k-1-i}\right) N=T^{-2} S$, that is, $T S N^{k-1}+$
$\cdots+T^{k-1} S N=0$. Postmultiplying the above equation by $N^{k-2}$, then $T S N^{k+k-3}+\cdots+T^{k-1} S N^{k-1}=0$, that is, $S N^{k-1}=0$. Through $T S N^{k-1}+\cdots+T^{k-1} S N=0$, we have $T^{2} S N^{k-2}+\cdots+T^{k-1} S N=0$. In the same way, we obtain $T^{k-1} S N=0$, that is, $S N=0$. From what has been discussed above, we have $S N=0$ if and only if $A^{\circledR} A^{D} A=A^{\circledR}$. Hence, Conditions (1) and (28) are equivalent.

By Lemma 1 and Lemma 4, we can get

$$
\begin{aligned}
A A^{D} & =U\left[\begin{array}{cc}
T & S \\
0 & N
\end{array}\right]\left[\begin{array}{cc}
T^{-1} & T^{-(k+1)} \widetilde{T} \\
0 & 0
\end{array}\right] U^{*} \\
& =U\left[\begin{array}{cc}
I_{\mathrm{rk}\left(A^{k}\right)} & T^{-k} \widetilde{T} \\
0 & 0
\end{array}\right] U^{*} .
\end{aligned}
$$

By applying [23] and (57), we have

$$
\begin{aligned}
A A^{D} A^{\circledR} & =U\left[\begin{array}{cc}
I_{\mathrm{rk}\left(A^{k}\right)} T^{-k} \widetilde{T} \\
0 & 0
\end{array}\right]\left[\begin{array}{cc}
T^{-1} & T^{-2} S \\
0 & 0
\end{array}\right] U^{*} \\
& =U\left[\begin{array}{cc}
T^{-1} & T^{-2} S \\
0 & 0
\end{array}\right] U^{*}=A^{\circledR} .
\end{aligned}
$$

Since the above formula, $A^{\circledR} A A^{D}=A^{\circledR} A^{D} A$ and $A^{\circledR} A$ $A^{D}=A^{\circledR}$ are equivalent to $A^{\circledR} A A^{D}=A A^{D} A^{\circledR}$. Thus, Conditions (1) and (29) are equivalent.

Because of [13] and (57), we can get

$$
\begin{aligned}
I-A A^{\oplus} & =U\left[\begin{array}{cc}
I_{\mathrm{rk}\left(A^{k}\right)} & 0 \\
0 & I_{n-\mathrm{rk}\left(A^{k}\right)}
\end{array}\right]\left[\begin{array}{cc}
I_{\mathrm{rk}}\left(A^{k}\right) & 0 \\
0 & 0
\end{array}\right] U^{*} \\
& =U\left[\begin{array}{lc}
0 & 0 \\
0 & I_{n-\mathrm{rk}\left(A^{k}\right)}
\end{array}\right] U^{*},
\end{aligned}
$$

$$
\begin{aligned}
A A^{D}\left(I-A A^{\oplus}\right) A= & U\left[\begin{array}{cc}
T^{k} & T^{-k} \widetilde{T} \\
0 & 0
\end{array}\right] \\
& \cdot\left[\begin{array}{cc}
0 & 0 \\
0 & I_{n-\mathrm{rk}\left(A^{k}\right)}
\end{array}\right]\left[\begin{array}{cc}
T & S \\
0 & N
\end{array}\right] U^{*} \\
= & U\left[\begin{array}{cc}
0 & T^{-k} \widetilde{T} N \\
0 & 0
\end{array}\right] U^{*} .
\end{aligned}
$$

If $S N=0$, then $A A^{D}\left(I-A A^{\oplus}\right) A=0$. Conversely, let $A A^{D}\left(I-A A^{\oplus}\right) A=0$, then $T^{-k}\left(\sum_{i=0}^{k-1} T^{i} S N^{k-1-i}\right) N=0$, that is, $T S N^{k-1}+\cdots+T^{k-1} S N=0$. By applying the method which is used to verify the equivalence of Conditions (1) and (28), we obtain $S N=0$. From what has been discussed above, $S N=0$ is equivalent to $A A^{D}\left(I-A A^{\oplus}\right) A=0$. Hence, Conditions (1) and (30) are equivalent.

From (59), we have

$$
\begin{aligned}
A^{k}\left(I-A A^{\oplus}\right) A & =U\left[\begin{array}{cc}
T^{k} & \widetilde{T} \\
0 & 0
\end{array}\right]\left[\begin{array}{cc}
0 & 0 \\
0 & I_{n-\mathrm{rk}\left(A^{k}\right)}
\end{array}\right]\left[\begin{array}{cc}
T & S \\
0 & N
\end{array}\right] U^{*} \\
& =U\left[\begin{array}{cc}
0 & \widetilde{T} N \\
0 & 0
\end{array}\right] U^{*} .
\end{aligned}
$$


If $S N=0$, then $A^{k}\left(I-A A^{\oplus}\right) A=0$. Conversely, let $A^{k}\left(I-A A^{\oplus}\right) A=0$, then $\left(\sum_{i=0}^{k-1} T^{i} S N^{k-1-i}\right) N=0$, that is, $T S N^{k-1}+\cdots+T^{k-1} S N=0$. By applying the method which is used to verify the equivalence of Conditions (1) and (28), we obtain $S N=0$. From what has been discussed above, $S N=0$ is equivalent to $A^{k}\left(I-A A^{\oplus}\right) A=0$. Hence, Conditions (1) and (31) are equivalent.

By using [13], (43), and (59), we can get

$$
\begin{aligned}
A A^{D, \dagger}\left(I-A A^{\oplus}\right) A= & U\left[\begin{array}{cc}
I_{\operatorname{rk}\left(A^{k}\right)} T^{-k} \widetilde{T} N N^{\oplus} \\
0 & 0
\end{array}\right] \\
& \cdot\left[\begin{array}{cc}
0 & 0 \\
0 & I_{n-\operatorname{rk}\left(A^{k}\right)}
\end{array}\right]\left[\begin{array}{cc}
T & S \\
0 & N
\end{array}\right] U^{*} \\
= & U\left[\begin{array}{cc}
0 & T^{-k} \widetilde{T} N \\
0 & 0
\end{array}\right] U^{*} .
\end{aligned}
$$

If $S N=0$, then $A A^{D, \dagger}\left(I-A A^{\oplus}\right) A=0$. Conversely, let $A A^{D, \dagger}\left(I-A A^{\oplus}\right) A=0$, then $T^{-k}\left(\sum_{i=0}^{k-1} T^{i} S N^{k-1-i}\right) N=0$, that is, $T S N^{k-1}+\cdots+T^{k-1} S N=0$. By applying the method which is used to verify the equivalence of Conditions (1) and (28), we obtain $S N=0$. From what has been discussed above, $S N=0$ is equivalent to $A A^{D, \dagger}\left(I-A A^{\oplus}\right) A=0$. Thus, Conditions (1) and (32) are equivalent.

Applying Lemma 4, [10], and (59), we obtain

$$
\begin{aligned}
A^{\dagger, D}\left(I-A A^{\oplus}\right) A= & U\left[\begin{array}{cc}
T^{*} \triangle & T^{*} \triangle T^{-k} \widetilde{T} \\
F_{N} S^{*} \triangle & F_{N} S^{*} \triangle T^{-k} \widetilde{T}
\end{array}\right] \\
& \cdot\left[\begin{array}{cc}
0 & 0 \\
0 & I_{n-\mathrm{rk}}\left(A^{k}\right)
\end{array}\right]\left[\begin{array}{cc}
T & S \\
0 & N
\end{array}\right] U^{*} \\
= & U\left[\begin{array}{cc}
0 & T^{*} \triangle T^{-k} \widetilde{T} N \\
0 & F_{N} S^{*} \triangle T^{-k} \widetilde{T} N
\end{array}\right] U^{*} .
\end{aligned}
$$

If $S N=0$, then $A^{\dagger, D}\left(I-A A^{\oplus}\right) A=0$. Conversely, let $A^{\dagger, D}\left(I-A A^{\oplus}\right) A=0$, then $T^{*}\left[T T^{*}+S F_{N} S^{*}\right]^{-1} T^{-k}\left(\sum_{i=0}^{k-1}\right.$ $\left.T^{i} S N^{k-1-i}\right) N=0$ and $F_{N} S^{*}\left[T T^{*}+S F_{N} S^{*}\right]^{-1} T^{-k}\left(\sum_{i=0}^{k-1}\right.$ $\left.T^{i} S N^{k-1-i}\right) N=0$. By using the method which is used to verify the equivalence of Conditions (1) and (28), we obtain $S N=0$. From what has been discussed above, $S N=0$ is equivalent to $A^{\dagger, D}\left(I-A A^{\oplus}\right) A=0$. Hence, Conditions (1) and (33) are equivalent.

By Lemma 4, [13], and (59), we have

$$
\begin{aligned}
A^{C, \dagger}\left(I-A A^{\oplus}\right) A= & U\left[\begin{array}{cc}
T^{*} \triangle & T^{*} \triangle T^{-k} \widetilde{T} N N^{\oplus} \\
F_{N} S^{*} \triangle & F_{N} S^{*} \triangle T^{-k} \widetilde{T} N N^{\oplus}
\end{array}\right] \\
& \cdot\left[\begin{array}{cc}
0 & 0 \\
0 & I_{n-\operatorname{rk}\left(A^{k}\right)}
\end{array}\right]\left[\begin{array}{cc}
T & S \\
0 & N
\end{array}\right] U^{*} \\
= & U\left[\begin{array}{cc}
0 & T^{*} \triangle T^{-k} \widetilde{T} N \\
0 & F_{N} S^{*} \triangle T^{-k} \widetilde{T} N
\end{array}\right] U^{*} .
\end{aligned}
$$

If $S N=0$, then $A^{C, \dagger}\left(I-A A^{\oplus}\right) A=0$. Conversely, let $A^{C, \dagger}\left(I-A A^{\oplus}\right) A=0$, then $T^{*}\left[T T^{*}+S F_{N} S^{*}\right]^{-1} T^{-k}\left(\sum_{i=0}^{k-1}\right.$ $\left.T^{i} S N^{k-1-i}\right) N=0$ and $F_{N} S^{*}\left[T T^{*}+S F_{N} S^{*}\right]^{-1} T^{-k}\left(\sum_{i=0}^{k-1}\right.$ $\left.T^{i} S N^{k-1-i}\right) N=0$. By applying the method which is used to verify the equivalence of Conditions (1) and (28), we obtain $S N=0$. From what has been discussed above, $S N=0$ is equivalent to $A^{C, \dagger}\left(I-A A^{\oplus}\right) A=0$. Hence, Conditions (1) and (34) are equivalent.

By applying $[4,8]$, we obtain $S N=0$ if and only if $A^{\oplus}$ $A^{2}=A^{2} A^{\mathrm{W}}$. Hence, Conditions (1) and (35) are equivalent.

Because of [16], (57) and

$$
\begin{aligned}
A A A^{D} & =U\left[\begin{array}{cc}
T & S \\
0 & N
\end{array}\right]\left[\begin{array}{cc}
I_{\operatorname{rk}\left(A^{k}\right)} & T^{-k} \widetilde{T} \\
0 & 0
\end{array}\right] U^{*} \\
& =U\left[\begin{array}{cc}
T & T^{-(k-1)} \widetilde{T} \\
0 & 0
\end{array}\right] U^{*} .
\end{aligned}
$$

If $S N=0$, then $A^{2} A^{D}=A A^{\oplus} A$. Conversely, let $A^{2} A^{D}=$ $A A^{\oplus} A$, then $T^{-(k-1)}\left(\sum_{i=0}^{k-1} T^{i} S N^{k-1-i}\right)=S$, that is, $T^{-(k-1)} S$ $N^{k-1}+\cdots+T^{-1} S N=0$. By applying the method which is used to verify the equivalence of Conditions (1) and (28), we obtain $S N=0$. From what has been discussed above, $S N=0$ is equivalent to $A^{2} A^{D}=A A^{\oplus} A$. Hence, Conditions (1) and (36) are equivalent.

\section{Generalized Cayley-Hamilton Theorem}

In this section, we extend the classical Cayley-Hamilton theorem to some special matrix such as the WG matrix.

Theorem 3 (see [21]). Let $A \in \mathbb{C}_{n, n} ;$ the characteristic polynomial of $A$ is

$$
p_{A}(s)=\operatorname{det}\left(s I_{n}-A\right)=s^{n}+a_{n-1} s^{n-1}+\cdots+a_{1} s+a_{0},
$$

then

$$
p_{A}(A)=A^{n}+a_{n-1} A^{n-1}+\cdots+a_{1} A+a_{0} I_{n}=0 .
$$

In [21], if $A$ is singular, then $a_{0}=0$.

Theorem 4. Let $A \in \mathbb{C}_{n, n}$ be singular with $\operatorname{Ind}(A)=k$. If

$$
\operatorname{det}\left(s I_{n}-A\right)=s^{n}+a_{n-1} s^{n-1}+\cdots+a_{1} s,
$$

then

$$
\begin{aligned}
A^{\circledR} & +a_{n-1}\left(A^{(\mathbb{1})}\right)^{2} \\
& +\cdots+a_{1}\left(A^{\mathbb{(}}\right)^{n}=0,
\end{aligned}
$$

where $A^{\circledR} \in \mathbb{C}_{n, n}$ is the weak group inverse of the matrix $A$.

Proof. Let $A \in \mathbb{C}_{n, n}$ be singular; we use the Cayley-Hamilton theorem, then

$$
A^{n}+a_{n-1} A^{n-1}+\cdots+a_{1} A=0 .
$$

Postmultiplying the above equation by $\left(A^{\circledR}\right)^{n+1}$, we get

$$
\begin{gathered}
A^{n}\left(A^{(}\right)^{n+1}+a_{n-1} A^{n-1}\left(A^{\circledR}\right)^{n+1} \\
+\cdots+a_{1} A\left(A^{(}\right)^{n+1}=0 .
\end{gathered}
$$


By using the properties of the WG matrix, that is, $A\left(A^{\circledR}\right)^{2}=A^{\circledR}$, we have $A\left(A^{\circledR}\right)^{n+1}=A\left(A^{\circledR}\right)^{2}\left(A^{\circledR}\right)^{n-1}=$ $A^{(\mathbb{1}}\left(A^{(\mathbb{1}}\right)^{n-1}=\left(A^{\circledR}\right)^{n}$. A similar method can be used to obtain $A^{2}\left(A^{\circledR}\right)^{n+1}=\left(A^{\circledR}\right)^{n-1}, \cdots, A^{n-1}\left(A^{\circledR}\right)^{n+1}=\left(A^{\circledR}\right)^{2}$, and $A^{n}\left(A^{\mathrm{W}}\right)^{n+1}=A^{\mathrm{W}}$. Substituting the above equations into (71), we have (69).

Example 1. Let

$$
A=\left[\begin{array}{cc}
1 & 0 \\
-1 & 0
\end{array}\right]
$$

It is easy to confirm that the weak group inverse $A^{\mathrm{W}} \in \mathbb{C}_{n, n}$ is

$$
A^{\circledR}=\left[\begin{array}{cc}
1 & 0 \\
-1 & 0
\end{array}\right] .
$$

Then,

$$
\operatorname{det}\left(s I_{2}-A\right)=\left|\begin{array}{cc}
s-1 & 0 \\
1 & s
\end{array}\right|=s^{2}-s
$$

From the classical Cayley-Hamilton theorem, we have

$$
A^{2}-A=\left[\begin{array}{cc}
1 & 0 \\
-1 & 0
\end{array}\right]^{2}-\left[\begin{array}{cc}
1 & 0 \\
-1 & 0
\end{array}\right]=\left[\begin{array}{ll}
0 & 0 \\
0 & 0
\end{array}\right] \text {. }
$$

By applying Theorem 4, we obtain

$$
\begin{aligned}
A^{\circledR}-\left(A^{\circledR}\right)^{2} & =\left[\begin{array}{cc}
1 & 0 \\
-1 & 0
\end{array}\right]-\left[\begin{array}{cc}
1 & 0 \\
-1 & 0
\end{array}\right]^{2} \\
& =\left[\begin{array}{ll}
0 & 0 \\
0 & 0
\end{array}\right] .
\end{aligned}
$$

Next, we extend the classical Cayley-Hamilton theorem to the WG inverse matrix. Let $A \in \mathbb{C}_{n, n}$ with $\operatorname{Ind}(A)=k$. From Lemma 1 and Lemma 2, it can be obtained

$$
\begin{gathered}
\operatorname{det}\left(s I_{n}-U\left[\begin{array}{cc}
T^{-1} & T^{-2} S \\
0 & 0
\end{array}\right] U^{*}\right) \\
=s^{n-r k\left(A^{k}\right)} \operatorname{rk}\left(s I_{\mathrm{rk}\left(A^{k}\right)}-T^{-1}\right) .
\end{gathered}
$$

The characteristic polynomial of $T^{-1}$ is

$$
\begin{aligned}
p_{T^{-1}}(s)= & \operatorname{det}\left(s I_{\mathrm{rk}\left(A^{k}\right)}-T^{-1}\right) \\
= & s^{\mathrm{rk}\left(A^{k}\right)}+b_{n-1} s^{\mathrm{rk}\left(A^{k}\right)-1}+\cdots \\
& +b_{n-\mathrm{rk}\left(A^{k}\right)+1} s+b_{n-\mathrm{rk}\left(A^{k}\right)} .
\end{aligned}
$$

It is given by the classical Cayley-Hamilton theorem

$$
\begin{aligned}
p_{T^{-1}}\left(T^{-1}\right)= & \left(T^{-1}\right)^{\mathrm{rk}\left(A^{k}\right)}+b_{n-1}\left(T^{-1}\right)^{\mathrm{rk}\left(A^{k}\right)-1} \\
& +\cdots+b_{n-\mathrm{rk}}\left(A^{k}\right)+1 \\
& T^{-1}+b_{n-\mathrm{rk}}\left(A^{k}\right) I_{\mathrm{rk}\left(A^{k}\right)}=0 .
\end{aligned}
$$

Postmultiplying the above equation by $T^{\mathrm{rk}\left(A^{k}\right)}$,

$$
\begin{aligned}
& I_{r k\left(A^{k}\right)}+b_{n-1} T+\cdots+b_{n-\mathrm{rk}\left(A^{k}\right)+1} T^{\mathrm{rk}\left(A^{k}\right)-1} \\
& \quad+b_{n-\mathrm{rk}\left(A^{k}\right)} T^{\mathrm{rk}\left(A^{k}\right)}=0 .
\end{aligned}
$$

By using (77) and (78), we have Theorem 5.

Theorem 5. Let $A \in \mathbb{C}_{n, n}$ with Ind $(A)=k$, then the characteristic polynomial of $A^{\mathbb{Q}}$ is

$$
\begin{aligned}
& p(s)=\operatorname{det}\left(s I_{n}-A\right)=s^{n}+b_{n-1} s^{n-1} \\
& +\cdots+b_{n-\mathrm{rk}\left(A^{k}\right)} s^{n-\mathrm{rk}\left(A^{k}\right),} \\
& \cdot\left(A^{\circledR}\right)^{n}+b_{n-1}\left(A^{\circledR}\right)^{n-1} \\
& +\cdots+b_{n-\mathrm{rk}\left(A^{k}\right)}\left(A^{\mathbb{1}}\right)^{n-\operatorname{rk}\left(A^{k}\right)}=0,
\end{aligned}
$$

where $b_{n-1}, \ldots, b_{n-r k\left(A^{k}\right)}$ is as in (78).

Example 2. Let

$$
A=\left[\begin{array}{llll}
1 & 0 & 1 & 0 \\
0 & 1 & 0 & 1 \\
0 & 0 & 0 & 1 \\
0 & 0 & 0 & 0
\end{array}\right],
$$

then $\operatorname{Ind}(A)=2, \operatorname{rk}\left(A^{2}\right)=2$, and

$$
\begin{aligned}
T & =\left[\begin{array}{ll}
1 & 0 \\
0 & 1
\end{array}\right], \\
T^{-1} & =\left[\begin{array}{ll}
1 & 0 \\
0 & 1
\end{array}\right], \\
A^{\mathbb{1}} & =\left[\begin{array}{llll}
1 & 0 & 1 & 0 \\
0 & 1 & 0 & 1 \\
0 & 0 & 0 & 0 \\
0 & 0 & 0 & 0
\end{array}\right] .
\end{aligned}
$$

Furthermore,

$$
\begin{aligned}
p_{T^{-1}}(s)= & s^{2}-2 s+1, p_{A}(s) \\
= & s^{2}\left(s^{2}-2 s+1\right)=s^{4}-2 s^{3}+s^{2} \\
& \cdot\left[\begin{array}{llll}
1 & 0 & 1 & 0 \\
0 & 1 & 0 & 1 \\
0 & 0 & 0 & 0 \\
0 & 0 & 0 & 0
\end{array}\right]^{4}-2\left[\begin{array}{llll}
1 & 0 & 1 & 0 \\
0 & 1 & 0 & 1 \\
0 & 0 & 0 & 0 \\
0 & 0 & 0 & 0
\end{array}\right]^{3}+\left[\begin{array}{llll}
1 & 0 & 1 & 0 \\
0 & 1 & 0 & 1 \\
0 & 0 & 0 & 0 \\
0 & 0 & 0 & 0
\end{array}\right]^{2} \\
= & 0 .
\end{aligned}
$$

\section{Conclusion}

In this paper, we give some new characterizations of the WG matrix. Some relations between various well-known generalized inverses and the WG matrix are proved, and 
generalized Cayley-Hamilton theorem for special matrices such as WG matrix is given. We are convinced that researches about the WG matrix will also gain more attention in the near future. Some perspectives for further researches can be described as follows: (1) our further goal will be to investigate the definition, properties, and characterizations of tensor WG matrix; (2) further, we will study the iterative algorithm of the tensor WG matrix and its application [28].

\section{Data Availability}

No data were used to support this study.

\section{Conflicts of Interest}

The authors declare that they have no conflicts of interest.

\section{Acknowledgments}

This work was supported partially by the Innovation Project of Guangxi Graduate Education (grant no. YCSW2021146), the Special Fund for Bagui Scholars of Guangxi (grant no. 2016A17), Guangxi Natural Science Foundation (grant no. 2018GXNSFAA138181), and the Special Fund for Science and Technological Bases and Talents of Guangxi (grant no. GUIKE AD19245148).

\section{References}

[1] S. L. Campbell and C. D. Meyer, Generalized Inverses of Linear Transformations, Society for Industrial and Applied Mathematics, Philadelphia, PA, USA, 2009.

[2] G. R. Wang, Y. M. Wei, and S. Z. Qiao, Generalized Inverses: Theory and Computations, Springer, Singapore, Singapore, 2018.

[3] O. M. Baksalary and G. Trenkler, "Core inverse of matrices," Linear and Multilinear Algebra, vol. 58, no. 6, pp. 681-697, 2010.

[4] K. Manjunatha Prasad and K. S. Mohana, "Core-EP inverse," Linear and Multilinear Algebra, vol. 62, no. 6, pp. 792-802, 2014.

[5] O. M. Baksalary and G. Trenkler, "On a generalized core inverse," Applied Mathematics and Computation, vol. 236, pp. 450-457, 2014.

[6] S. B. Malik and N. Thome, "On a new generalized inverse for matrices of an arbitrary index," Applied Mathematics and Computation, vol. 226, pp. 575-580, 2014.

[7] M. Mehdipour and A. Salemi, "On a new generalized inverse of matrices," Linear and Multilinear Algebra, vol. 66, no. 5, pp. 1046-1053, 2018.

[8] H. Wang and J. Chen, "Weak group inverse," Open Mathematics, vol. 16, no. 1, pp. 1218-1232, 2018.

[9] D. E. Ferreyra, V. Orquera, and N. Thome, "A weak group inverse for rectangular matrices," Revista de la Real Academia de Ciencias Exactas, Físicas y Naturales. Serie A. Matemáticas, vol. 113, no. 4, pp. 3727-3740, 2019.

[10] D. Mosić and D. C. Zhang, "Weighted weak group inverse for Hilbert space operators," Frontiers of Mathematics in China, vol. 15, no. 4, pp. 709-726, 2020.

[11] M. Zhou, J. Chen, and Y. Zhou, "Weak group inverses in proper *-rings," Journal of Algebra and Its Applications, vol. 19, no. 12, Article ID 2050238, 2019.
[12] Y. Zhou, J. Chen, and M. Zhou, "m-weak group inverses in a ring with involution," Revista de la Real Academia de Ciencias Exactas, Físicas y Naturales. Serie A. Matemáticas, vol. 115, no. 1, p. 2, 2020.

[13] D. Mosić and P. S. Stanimirovi, "Representations for the weak group inverse," Applied Mathematics and Computation, vol. 397, no. 6, Article ID 125957, 2021.

[14] H. Wang and X. Liu, "The weak group matrix," Aequationes Mathematicae, vol. 93, no. 6, pp. 1261-1273, 2019.

[15] H. Yan, H. X. Wang, H. Wang, K. Zuo, and Y. Chen, "Further characterizations of the weak group inverse of matrices and the weak group matrix," AIMS Mathematics, vol. 6, no. 9, pp. 9322-9341, 2021.

[16] H. Wang, "Core-EP decomposition and its applications," Linear Algebra and Its Applications, vol. 508, pp. 289-300, 2016.

[17] C. Y. Deng and H. K. Du, "Representations of the moorepenrose inverse of $2 \times 2$ block operator valued matrices," Journal of the Korean Mathematical Society, vol. 46, no. 6, pp. 1139-1150, 2009.

[18] D. E. Ferreyra, F. E. Levis, and N. Thome, "Characterizations of k-commutative equalities for some outer generalized inverses," Linear and Multilinear Algebra, vol. 68, no. 1, pp. 177-192, 2018.

[19] W. Jiang, K. Z. Zuo, and K. Zuo, "Revisiting of the BT-inverse of matrices," AIMS Mathematics, vol. 6, no. 3, pp. 2607-2622, 2021.

[20] K. Zuo and Y. Cheng, "The new revisitation of core EP inverse of matrices," Filomat, vol. 33, no. 10, pp. 3061-3072, 2019.

[21] R. A. Horn and C. R. Johnson, Matrix Analysis, Cambridge University Press, Cambridge, UK, 2012.

[22] F. R. Chang and H. C. Chen, "The generalized CayleyHamilton theorem for standard pencils," Systems \& Control Letters, vol. 18, no. 3, pp. 179-182, 1992.

[23] T. Kaczorek, "An extension of the Cayley-Hamilton theorem for a standard pair of block matrices," Applied Mathematics and Computation Science, vol. 8, pp. 511-516, 1998.

[24] T. Kaczorek, "Extension of the Cayley-Hamilton theorem to continuous-time systems with delays," International Journal of Applied Mathematics and Computer Science, vol. 15, no. 2, pp. 231-234, 2005.

[25] T. Kaczorek, Selected Problems of Fractional Systems Theory, Springer, Berlin, Germany, 2012.

[26] H. X. Wang, J. L. Chen, and G. J. Yan, "Generalized CayleyHamilton theorem for core-EP inverse matrix and DMP inverse matrix," Journal of Southeast University (English Edition), vol. 34, no. 1, pp. 135-138, 2018.

[27] T. Kaczorek, "Cayley-Hamilton theorem for Drazin inverse matrix and standard inverse matrices," Bulletin of the Polish Academy of Sciences, Technical Sciences, vol. 64, no. 4, pp. 793-797, 2016.

[28] S. K. Mitra, P. Bhimasankaram, and S. B. Malik, Matrix Partial Orders, Shorted Operators and Applications, World Scientific, Singapore, Singapore, 2010. 